\title{
Thrombocytopenia induced by a taurine-containing energy drink: an adverse reaction to herbal medicine
}

\author{
Federico Pasin, ${ }^{1}$ Emanuela Porro, ${ }^{2}$ Francesco Frattini, ${ }^{3}$ Pierpaolo Vescovi, ${ }^{3}$ Massimo Franchini, ${ }^{4}$ Paolo Sansoni ${ }^{2}$ \\ ${ }^{1}$ Department of General Medicine, Hospital Institutes of Cremona, Cremona; ${ }^{2}$ Department of Clinical and Experimental Med- \\ icine, University of Parma, Parma; ${ }^{3}$ Medical Department, AO Carlo Poma, Mantova; ${ }^{4}$ Department of Laboratory Medicine, \\ AO Carlo Poma, Mantova, Italy
}

\begin{abstract}
Thrombocytopenia is a well-recognized adverse effect of many drugs. The association of thrombocytopenia with herbal remedies, nutritional supplements, foods and beverages, complementary or alternative medicines, has been rarely described. There are reports of thrombocytopenia caused by quinine-containing beverages, cow's milk, cranberry juice, Jui, a Chinese herbal tea, Lupinus termis bean and tahini. A definite evidence of a causal association with thrombocytopenia is warranted; nevertheless not always there is provided probable or possible evidence in the association with thrombocytopenia. We report the first case, to our knowledge, of thrombocytopenia induced by taurine, present in an energy drink prescribed to our patient as tonic treatment.
\end{abstract}

\section{Introduction}

Thrombocytopenia, defined as a platelet count of less than $100,000 \mathrm{~mm}^{3}$, is a pathologic condition caused by different mechanisms and with fatal consequences. ${ }^{1}$ According to the literature, drug-induced thrombocytopenia is very often associated with the use of non-steroidal anti-inflammatory drugs, sulfonamides, cinchona alkaloids (derivatives of chinine and chinidine), thiazide containing diuretics, penicillin, gold salts, sulfosalazine and heparin, although the actual list of compounds is more extended ${ }^{2-4}$ and more than 1400 drugs have been implicated in thrombocytopenia (at least one case report). Interestingly, in only 67 of such cases, specific analyses have established

Correspondence: Federico Pasin, Department of General Medicine, Hospital Institutes of Cremona, viale Concordia 1, 26100 Cremona, Italy.

Tel.: +39.0375.281459.

E-mail: f.pasin@ospedale.cremona.it

Key words: thrombocytopenia, drug-induced thrombocytopenia, herbal remedy, complementary/alternative medicine, nutritional supplement.

Received for publication: 20 December 2013

Accepted for publication: 7 February 2014.

This work is licensed under a Creative Commons Attribution NonCommercial 3.0 License (CC BY-NC 3.0).

CCopyright F. Pasin et al., 2014

Licensee PAGEPress, Italy

Italian Journal of Medicine 2014; 8:259-261

doi:10.4081/itjm.2014.466 the direct involvement of the drug as a causative factor of the disease. ${ }^{5}$ Conversely, many chemotherapeutic agents are known to induce thrombocytopenia by direct inhibition of megakaryopoiesis.

Epidemiologic studies indicate that the incidence of drug-induced thrombocytopenia in the general population is about 10 cases per one million inhabitants per year. In specific populations such as in hospitalized patients affected by tumors, who undergo chemotherapy, the incidence is considerably higher. Even though druginduced thrombocytopenia is a relatively rare adverse drug reaction, its consequences can be severe. ${ }^{6-8}$

A reduced platelet count can be the result of various mechanisms: impaired production by the bone marrow, accelerated peripheral degradation and increased hemocatheresis by the spleen. To understand the aetiology of thrombocytopenia, the following tests are usually carried out: a careful clinical and pharmacological history, physical examination, peripheral blood tests including laboratory measurements of immature platelet fraction (IPF), evaluation of the presence of anti-platelet antibodies, diagnostic imaging and lastly the evaluation of bone marrow morphology by aspiration or biopsy.

Immune thrombocytopenic purpura (ITP) is an acquired immune disorder. The diagnosis is based on the finding of an isolated reduced platelet count in the absence of other hematologic abnormalities or clinical evidence of concomitant pathologic states. While the presence of anti-platelet antibodies may strongly suggest the diagnosis of ITP, a high percentage of cases are serum negative.

The pathogenic mechanism underlying ITP is the immune-mediated destruction of platelets by specific IG against epitopes expressed on their surface. . $^{8-10}$ These epitopes are frequently glycoproteins, such as 
GPIIbIIIa. The antigenic mimetism, the possible cross-reaction of antibodies against viral or bacterial epitopes, and the alterations of the immune system (such as the loss of peripheral tolerance) are further determining events of this autoimmune defects. ${ }^{12,13}$

The development of ITP is triggered or favored by previous viral [HIV, hepatitis $\mathrm{C}$ virus (HCV), varicellazoster virus] or bacterial (Helicobacter pylori) infections and exposure to drugs or chemical substances.

In this study, we report a case of thrombocytopenia induced by taurine, an antioxidant and energizing substance commonly present in high-energy drinks used as tonic treatment. To our knowledge, this is the first case reported in the literature.

\section{Case Report}

An 85-year old woman in overall good health conditions was admitted to our Department because of sudden development of bilateral pre-tibial petechiae and large ecchymoses on the trunk and limbs. The patient was under anticoagulants treatment because of chronic atrial fibrillation and mitral valve biologic prosthesis, implanted ten years earlier. Therefore, prothrombin time and international normalized ratio (INR) values were controlled and resulted in the normal therapeutic range (INR: 2-2.5). In contrast, serial haemocytometric tests documented severe thrombocytopenia (platelets count $<10,000 \mathrm{~mm}^{3}$ ). Previous laboratory analyses, performed 20 days earlier, showed normal platelets count $\left(230,000 \mathrm{~mm}^{3}\right)$. The clinical history of the patient excluded recent viral or bacterial infection or changes in the usual pharmacologic therapy (digoxin, calcium channel blockers, diuretics and warfarin). Physical examination showed, in addition to both extremities, also petechiae on the mouth and conjunctival mucosa. Cardiopulmonary performance was within normal limits.

Morphologic examination of peripheral blood showed rare giant platelets. No alterations were observed in white and red cell counts, excluding the presence of hematologic diseases. An increased value of IPF was detected (19\% n.v. 1\%-3\%) indicating a compensatory response of the bone marrow. In addition, IPF can be considered an indirect index of peripheral platelets destruction. Total complement, $\mathrm{C} 3$ and $\mathrm{C} 4$ were within normal limits. The search for antiplatelets antibodies, autoimmunity, HIV, HCV and all serological and microbiological tests including antistreptolysin value were within the normal range.

Renal, hepatic, thyroid and endocrine-metabolic functions were normal. An ultrasonography of the abdomen showed normal volumes of the liver and spleen. Finally an echocardiography study showed concentric hypertrophy with normal ejection fraction (55\%), normal function and morphology of mitral valve biologic prosthesis with moderate right side chamber dilation. These clinical and laboratory data led us to look for possible recent causes of the sudden development of thrombocytopenia. A careful investigation gave us the opportunity to discover an energy drug, prescribed by the family physician, as the likely cause triggering the platelet disease. In fact, the patient had assumed, during the week preceding hospitalization, a preparation containing guarana (a caffeine-containing alkaloid), royal jelly, high amounts of taurine (4.5\%), eleutherococcus and other herbal remedies. The withdrawal of the energy drug, together with an immunosuppressive dose of methyl-prednisolone ( $1 \mathrm{mg} / \mathrm{kg} /$ day $)$ produced a rapid and progressive rise of platelets count along with the progressive regression of the clinical manifestations. The platelet count returned to the normal value in less than 2 weeks with persistent normal count $(>180,000$ $\mathrm{mm}^{3}$ ) even at the last follow up, 1 year after the onset of thrombocytopenia.

\section{Discussion}

There are two mechanisms underlying the production of drug-induced antibodies, one is the constitution of steady covalent binding between a drug component and the external surface of the platelets, which in turn induces the development of a immunogenic neo-antigen; the other is represented by modifications of platelet membrane glycoproteins (such as GPIIbIIIa, GPIbIX, PECAM-1), ${ }^{14}$ which increase their immunogenic activity. In addition, a variety of studies have shown that the immunologic activity of the drugs is specifically due to their single active pharmacologic components; in fact, cross-reactivity is absent even between thrombocytopenic drugs, such as quinidine and sulfamethoxazole. ${ }^{11,12,15}$ The latter is one of the most frequent drug implicated in thrombocytopenia; it belongs to the class of sulfonamides, which also include sulpha-drugs. ${ }^{16}$ The chemical structure of these compounds contains a group constituted by an atom of sulfur with valence 6 and double oxidation $\left(\mathrm{S}-\mathrm{O}_{2}\right)$ and a link with an amino-nitrogen atom $\left(\mathrm{NH}_{2}\right)$; their general formula is $\mathrm{R}-\mathrm{SO}_{2}-\mathrm{NH}_{2}$, with $\mathrm{R}$ representing an aromatic group. Intriguingly, the general chemical structure of taurine, which is also named 2-aminoetanosulphonic acid, mimics that of sulfonamides and contains a functional group of hydroxylated sulfonic acid, linked to an amino-nitrogen atom; its general formula is $\mathrm{OH}-\mathrm{SO}_{2}-\mathrm{NH}_{2}$.

Examination of the scientific literature failed to provide evidence of similar cases of thrombocytopenia induced by energy drugs. However, in the light of the mechanisms underlying drug-induced platelets destruction, the analogy between chemical structures of drugs known to be most often responsible of thrombocytopenia (i.e. quinine, sulfonamides, gold salts, etc.) and those present in the energy mixture used by our patient, sug- 
gests that the same chemicals were responsible for the disease. Thus, the analogy with sulfonamides structures allowed us to advance the hypothesis that our patient represents a case of taurine induced thrombocytopenia. Unfortunately, the assay of antibodies versus hapten-glycoprotein complex/sulfonamide-like group in the serum of our patient was not possible, because is available only in high specialized laboratories. Thus, the similarities in biochemical structure between sulfonamide and taurine, the time course correlation between ingestion of this compound, the development of thrombocytopenia and finally, the persistent normal platelet count after withdrawal of causative drug, strongly support our conclusion. However, further laboratory studies are needed to corroborate our hypothesis.

\section{Conclusions}

According to others previously reported clinical cases, a deep investigation about the consumption of herbal remedies or other unconventional medicine, including energy drinks, ${ }^{17}$ is mandatory to rule out or confirm a possible adverse event including immunemediated reaction; ${ }^{18-20}$ all physicians could contribute to increase the knowledge on this field of medical science.

\section{References}

1. George JN, Woolf SH, Raskob GE, et al. Idiopathic thrombocytopenic purpura: a practice guideline developed by explicit methods for The American Society of Hematology. Blood 1996;88:3-40.

2. George JN, Raskob GE, Shah SR, et al. Drug-induced thrombocytopenia: a systematic review of published case reports. Ann Intern Med 1998;129:886-90.

3. Rizvi MA, Kojouri K, George JN. Drug-induced thrombo-cytopenia: an updated systematic review [letter]. Ann Intern Med 2001;134:346.

4. Aster RH, Bougie DW. Drug-induced immune thrombocytopenia. N Eng J Med 2007;357:580-7.

5. Hibbard AB, Medina PJ, Vesely SK. Reports of drug-in- duced thrombocytopenia [letter]. Ann Intern Med 2003; 138:239.

6. Pedersen-Bjergaard U, Andersen M, Hansen PB. Druginduced thrombocytopenia: clinical data on 309 cases and the effect of corticosteroid therapy. Eur J Clin Pharmacol 1997;52:183-9.

7. Danielson DA, Douglas SW $3^{\text {rd }}$, Herzog P, et al. Druginduced blood disorders. JAMA 1984;252:3257-60.

8. Kaufman DW, Kelly JP, Johannes CB, et al. Acute thrombo-cytopenic purpura in relation to the use of drugs. Blood 1993;82:2714-8.

9. Aster RH. Can drugs cause autoimmune thrombocytopenic purpura? Semin Hematol 2000;37:229-38.

10. Aster RH. Drug-induced immune thrombocytopenia: an overview of pathogenesis. Semin Hematol 1999;36:2-6.

11. Aljitawi OS, Krishnan K, Curtis BR, et al. Serologically documented loracarbef (Lorabid)-induced immune thrombocytopenia. Am J Hematol 2003;73:41-3.

12. Burgess JK, Lopez JA, Berndt MC, et al. Quinine-dependent antibodies bind a restricted set of epitopes on the glycoprotein Ib-IX complex: characterization of the epitopes. Blood 1998;92:2366-73.

13. Burgess JK. Molecular mechanisms of drug-induced thrombocytopenia. Curr Opin Hematol 2001;8:294-8.

14. Kroll H, Sun QH, Santoso S. Platelet endothelial cell adhesion molecule-1 (PECAM-1) is a target glycoprotein in drug-induced thrombocytopenia. Blood 2000;96: 1409-14.

15. Wazny LD, Ariano RE. Evaluation and management of drug-induced thrombocytopenia in the acutely ill patient. Pharmacotherapy 2000;20:292-307.

16. Frisch JM. Clinical experience with adverse reactions to trimethoprim-sulfamethoxazole. J Infect Dis 1973;128: S607-11.

17. Clauson KA, Shields KM, McQueen CE, Persad N. Safety issues associated with commercially available energy drinks. J Am Pharm Assoc 2008;48:e55-67.

18. Menniti-Ippolito F, Mozzanti G, Santuccio C, et al. Surveillance of suspected aderse reactions to natural health products in Italy. Pharmacoepidemiol Drug Saf 2008; 17:626-35

19. Wolk BJ, Ganetsky M, Babu KM. Toxicity of energy drinks. Curr Opin Pediatr 2012;24:243-51.

20. Royer DJ, George JN, Terrell DR. Thrombocytopenia as an adverse effect of complemetary and alternative medicines, herbal remedies, nutritional supplements, foods, and beverages. Eur J Haematol 2010;84:421-9. 